\title{
Classification of Uniparental Isodisomy Patterns That Cause Autosomal Recessive Disorders: Proposed Mechanisms of Different Proportions and Parental Origin in Each Pattern
}

\author{
Yo Niida ${ }^{a}$ Mamoru Ozakia, b Masaki Shimizuc Kazuyuki Uenoc \\ Tomomi Tanaka ${ }^{d}$ \\ ${ }^{a}$ Center for Clinical Genomics, Kanazawa Medical University Hospital, ${ }^{b}$ Division of Genomic Medicine, Department \\ of Advanced Medicine, Medical Research Institute, Kanazawa Medical University, Uchinada, 'Department of \\ Pediatrics, School of Medicine, Institute of Medical, Pharmaceutical and Health Sciences, Kanazawa University, \\ Kanazawa, and ${ }^{\mathrm{d}}$ Department of Pediatrics, Faculty of Medicine, University of Toyama, Toyama, Japan
}

\section{Keywords}

Autosomal recessive disorders · Genetic counselling · Monosomy rescue $\cdot$ SNP array $\cdot$ Trisomy rescue $\cdot$ Uniparental isodisomy

\begin{abstract}
Patients with autosomal recessive (AR) disorders are usually born to parents both of whom are heterozygous carriers of the disease. However, in some instances only one of the parents is a carrier and a mutation is segregated to the patient through uniparental isodisomy (UPiD). Recently, an increasing number of such case reports has been published, and it has become clear that there are several different UPiD patterns that cause AR disorders. In this article, we report 3 remarkable patients with different patterns of UPiD. We then review 85 cases collected in the literature. We realized that they can be classified into 3 patterns: UPiD of the whole chromosome, segmental UPiD with uniparental heterodisomy (UPhD), and segmental UPiD caused by post-zygotic mitotic
\end{abstract}

\section{KARGER}

(C) 2018 S. Karger AG, Basel

E-Mail karger@karger.com

www.karger.com/cgr recombination (MiRe). Whole chromosomal UPiD accounted for the majority of cases, with paternal origin accounting for approximately twice as many cases as maternal origin. Most cases of segmental UPiD with UPhD were of maternal origin, with a dominancy of nondisjunction in meiosis I, while segmental UPiD through MiRe is the smallest pattern with equal parental origin. These differences in proportion and parental origin in each pattern can be explained by considering nondisjunction during oogenesis as the starting point and UPiD as subsequent events.

(c) 2018 S. Karger AG, Basel

Patients with autosomal recessive (AR) inherited disorders are usually born to parents both of whom are heterozygous carriers of the disease-causing mutation. However, in some instances, AR disorders are caused by only one of the parents being a carrier and the mutation allele had then segregated to the patient through uniparental disomy (UPD). Distinguishing these 2 mechanisms is 
very important for the practice of clinical genetics in 2 respects. First, since UPD is basically a rare accidental error of chromosomal segregation during gametogenesis or early fertilization, the recurrence risk for siblings is not $25 \%$ as in general AR diseases, but it can rather be negligible in these families. Second, there is a possibility for the patient to develop symptoms due to imprinting genes located on the UPD chromosome in addition to those caused by the AR disease.

In order to correctly understand this phenomenon, it is important to know that there are several patterns of UPD [Liehr, 2010; Lapunzina and Monk, 2011] and not all of them necessarily cause AR disorders. UPD is classified into uniparental isodisomy (UPiD) and uniparental heterodisomy (UPhD). UPiD refers to the inheritance of 2 copies of the same chromosome from 1 parent, while UPhD describes the inheritance of both homologous chromosomes from 1 parental pair. Although imprinting disorders are caused by both types of UPD, AR disorders are caused by UPiD only. Additionally, UPiD and UPhD chromosomal segments are sometimes mixed on the same chromosome, and the development of AR disorders depends on whether the responsible mutated gene is present in an UPiD segment or not. These cases of AR disorders caused by UPiD have been recognized as a rare exceptional event. However, recently there have been an increasing number of case reports, and it has become clear that there are several UPiD patterns that cause AR disorders. Furthermore, most of them are precisely distinguished by analyzing both the patient and parents using high-density microsatellite analysis or, more recently, by using single nucleotide polymorphism (SNP) DNA micro arrays (online suppl. Table 1; see www.karger.com/doi/10.1159/000488572 for all online suppl. material).

Here, we present 3 demonstrative patients with different patterns of UPiD. We then review the literature of AR disorders caused by UPiD and classify them by UPiD patterns. We show that there are 3 major patterns of this phenomenon and that the ratio of the parental origin of each pattern is clearly different. Finally, we propose a possible model to explain these differences.

\section{Case Report}

Patient 1

A 2-year-old girl with nemaline myopathy 2 (OMIM \#256030) was referred to our hospital for genetic counselling and detailed molecular examination of the family. She was diagnosed in a former hospital by gene panel testing using target capture/next-gen- eration sequencing for neuromuscular diseases [Tian et al., 2015]. A nonsense mutation in NEB, NM_001164508.1:c.24681C > G, NP_00115980.1:p.(Tyr8227*), was detected in the patient and seemed to be in a homozygous state concordant with an AR inheritance. However, this mutation was detected only in her father, who was a heterozygous carrier, but not in her mother (Fig. $1 \mathrm{~A})$. To interpret this result, 2 possibilities were considered: (1) that the maternal mutation was the result of a gene deletion and (2) that the patient developed the disease through paternal UPiD. To confirm these possibilities, we performed a SNP array analysis.

\section{Patient 2}

Patient 2 was born to non-consanguineous Japanese parents at 37 gestational weeks by natural vaginal delivery with a birth weight of 2,346 $\mathrm{g}(-0.89 \mathrm{SD})$. After birth, he showed tachypnea (60 70 breaths per minute) and poor weight gain $(2,302 \mathrm{~g}$ at 14 days of age). Arterial blood gas analysis indicated metabolic acidosis with a normal anion gap of $13.6(12 \sim 14) \mathrm{mEq} / \mathrm{L}, \mathrm{pH} 7.139, \mathrm{pCO}_{2} 19.7$ $\mathrm{mmHg}, \mathrm{pO}_{2} 74.2 \mathrm{mmHg}, \mathrm{HCO}_{3}{ }^{-} 6.4 \mathrm{mEq} / \mathrm{L}, \mathrm{BE}-21.5 \mathrm{mmol} / \mathrm{L}$, $\mathrm{Na} 142 \mathrm{mEq} / \mathrm{L}, \mathrm{K} 3.8 \mathrm{mEq} / \mathrm{L}, \mathrm{Cl} 122 \mathrm{mEq} / \mathrm{L}$. Since urine $\mathrm{pH}$ was 7.0 (>5.5), he was diagnosed as having distal (type I) renal tubular acidosis (RTA) [Sharma et al., 2015] and was treated by intravenous administration of sodium bicarbonate. The metabolic acidosis was then improved and maintained by oral administration of a potassium citrate-sodium citrate hydrate mixture. Serum calcium and thyroid hormone levels were normal, and there were no other secondary causes of RTA throughout the period of investigation. Accordingly, a genetic cause of his congenital and primary RTA was suspected [Batlle et al., 2001], and we investigated 2 genes, ATPV0A4 for RTA, distal, autosomal recessive (OMIM \#602722) and $A T P 6 V 1 B 1$ for RTA, distal, with progressive nerve deafness (OMIM \#267300).

\section{Patient 3}

The patient was a 1-year-old boy with Prader-Willi syndrome. This case was not the result of an AR disorder, but it is remarkable and important in understanding the mechanism of segmental $\mathrm{UPiD}$ with UPhD and is therefore presented here. A 40-year-old woman was subjected to fetal chromosomal analysis by chorionic villus sampling (CVS) due to high maternal age. The result showed $47, \mathrm{XY},+15$ and the fetal chromosome analysis was therefore reexamined by amniocentesis. The result was $46, \mathrm{XY}$ and it was considered that the CVS karyotype was caused by confined placental mosaicism. The pregnancy was continued, and a boy weighing $1,628 \mathrm{~g}(-2.92 \mathrm{SD})$ was born at 36 weeks of gestation. After birth, his developmental milestones were delayed. Feeding difficulty, muscular hypotonia, and minor anomalies, including small hands, almond shaped eyes, narrow forehead and undescended testis with micropenis, suggested Prader-Willi syndrome. To confirm the diagnosis, he was referred to our hospital for molecular and cytogenetic analysis.

\section{Material and Methods}

The gene mutations were screened by CHIPS (CEL nuclease mediated heteroduplex incision with polyacrylamide gel electrophoresis and silver staining) technology [Niida et al., 2012a, 2015]
138

Cytogenet Genome Res 2018;154:137-146 DOI: $10.1159 / 000488572$
Niida/Ozaki/Shimizu/Ueno/Tanaka 
Fig. 1. Direct sequencing results of $N E B$ in patient $1(\mathbf{A})$ and ATP6V0A4 in patient 2 (B). In both cases, only one of the parents was a heterozygous carrier and the patients had homozygous mutations. C SNRPN methylation test of patient 3. A faint band corresponding to the unmethylated form is seen in the patient. AS, Angelman syndrome; L, 100 bp ladder, M, methylated; PWS, Prader-Willi syndrome; U, unmethylated.

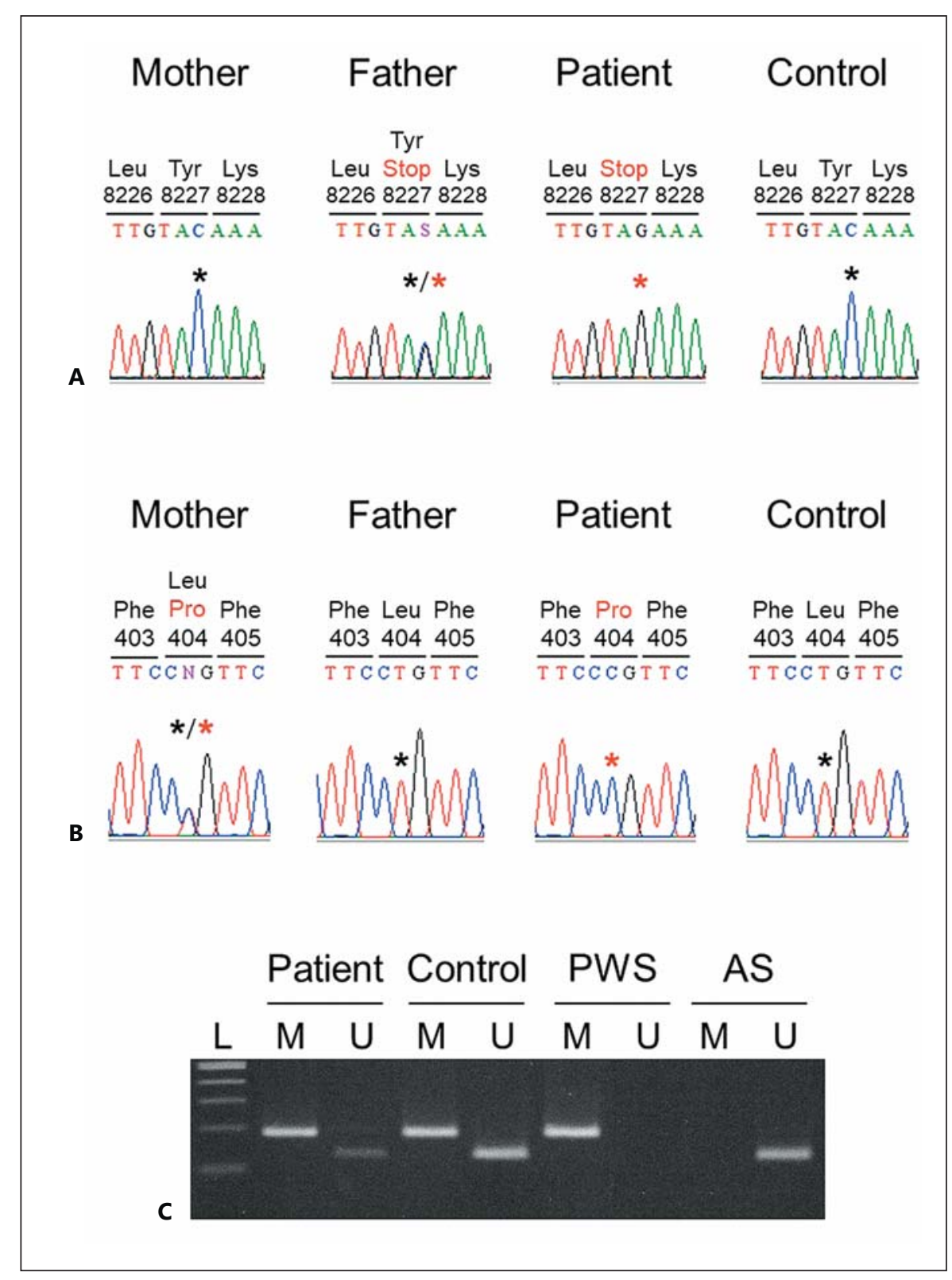

and confirmed by direct sequencing with an ABI 3130xl Genetic Analyser and BigDye version 3.1 Cycle Sequencing Kit (Thermo Fisher Scientific). For patient 3, the SNRPN methylation test was performed according to an established protocol [Kosaki et al., 1997] with some modifications. Chromosomal analysis was conducted on trypsin and G-banded metaphases according to established guidelines. For patient 1, DNA microarray analysis was performed using a CytoScan 750K Array (Affymetrix) and analyzed using Chromosome Analysis Suite (ChAS) 2.1 Software (Affymetrix). Patients 2 and 3 were analyzed using the Genome-Wide Human Single Nucleotide Polymorphism (SNP) 6.0 Array (Affymetrix) with Genotyping Console Software (Affymetrix). In all cases, segments which included at least 5 consecutive probes and which were at least $50 \mathrm{~kb}$ in size were selected.

Uniparental Isodisomy Patterns that Cause Autosomal Recessive Disorders

\section{Results}

In a SNP array, several thousand SNPs are detected simultaneously for each chromosome. The analysis of both the parents and the patient makes it possible to determine how the components of the parental chromosomes are passed on to their child. Interpretations of each SNP and examples for each patient are shown in Tables 1 and 2.

\section{Patient 1}

SNP array revealed UPiD of the whole chromosome 2 in the patient (Fig. 2A). The copy number state was 2 over 
Table 1. Interpretation of SNP calls in trio analysis

\begin{tabular}{lllll}
\hline $\begin{array}{l}\text { Informative SNP } \\
\text { segregation }\end{array}$ & & $\begin{array}{l}\text { Paternal } \\
\text { allele }\end{array}$ & $\begin{array}{l}\text { Maternal } \\
\text { allele }\end{array}$ & $\begin{array}{l}\text { Patient } \\
\text { (child) allele }\end{array}$ \\
\hline $\begin{array}{l}\text { Heterozygous from } \\
\text { both parents }\end{array}$ & AA & BB & AB \\
\hline No maternal allele & $\begin{array}{l}\text { paternal UPiD } \\
\text { paternal UPiD or UPhD }\end{array}$ & $\begin{array}{l}\mathrm{AB} \\
\mathrm{AA}\end{array}$ & $\begin{array}{l}\mathrm{BB} \\
\mathrm{BB}\end{array}$ & $\begin{array}{l}\mathrm{AA} \\
\mathrm{AA}\end{array}$ \\
\hline No paternal allele & maternal UPiD & $\mathrm{AA}$ & $\mathrm{AB}$ & $\mathrm{BB}$ \\
& maternal UPiD or UPhD & $\mathrm{AA}$ & $\mathrm{BB}$ & $\mathrm{BB}$ \\
\hline
\end{tabular}

$\mathrm{UPhD}$, uniparental heterodisomy; UPiD, uniparental isodisomy.

Table 2. Examples for the segregation of SNP in the patients

\begin{tabular}{|c|c|c|c|c|c|c|}
\hline Probe Set ID & dbSNP RS ID & $\begin{array}{l}\text { Chromosomal } \\
\text { position }\end{array}$ & $\begin{array}{l}\text { Father } \\
\text { base calls }\end{array}$ & $\begin{array}{l}\text { Mother } \\
\text { base calls }\end{array}$ & $\begin{array}{l}\text { Patient } \\
\text { base calls }\end{array}$ & Interpretation \\
\hline \multicolumn{7}{|l|}{ Patient 1} \\
\hline S-3OCCW & rs10171046 & $14,505,949$ & GT & GG & TT & paternal UPiD \\
\hline S-3XJJR & rs17339549 & $80,078,045$ & $\mathrm{AG}$ & GG & AA & paternal UPiD \\
\hline \multicolumn{7}{|l|}{ Patient 2} \\
\hline SNP_A-2235892 & rs17144537 & $8,228,716$ & GG & AA & AG & heterozygous from both parents \\
\hline SNP_A-2066458 & rs3807827 & $8,230,591$ & AA & GG & AG & heterozygous from both parents \\
\hline SNP_A-8606744 & rs13244158 & $67,291,581$ & TT & $\mathrm{TC}$ & $\mathrm{CC}$ & maternal UPiD \\
\hline SNP_A-8585105 & rs12669455 & $67,313,438$ & GG & AG & AA & maternal UPiD \\
\hline \multicolumn{7}{|l|}{ Patient 3} \\
\hline SNP_A-1794253 & rs4405519 & $22,777,875$ & AA & GG & GG & maternal UPiD or UPhD \\
\hline SNP_A-8387354 & rs8028366 & $25,076,893$ & AA & $\mathrm{TA}$ & TA & patient is heterozygous ${ }^{\mathrm{a}}$ \\
\hline SNP_A-4298692 & rs4028400 & $25,078,589$ & GG & AA & AA & maternal UPiD or UPhD ${ }^{a}$ \\
\hline SNP_A-8649732 & rs2727082 & $66,081,311$ & TT & CT & $\mathrm{CC}$ & maternal UPiD \\
\hline
\end{tabular}

UPhD, uniparental heterodisomy; UPiD, uniparental isodisomy. ${ }^{\text {a }}$ Since 2 adjacent probes indicate heterozygous and maternal UPD, it is presumed to be maternal UPhD.

the entire chromosome, including the $N E B$ locus. In addition, SNP probes covering the entire chromosome 2 showed AA or BB (means homozygous) but not $\mathrm{AB}$ (heterozygous), indicating that the 2 copies of chromosome 2 in the patient came from the same origin and were duplicated, i.e., UPiD of the whole chromosome. It was therefore concluded that the NEB mutation, c.24681C $>\mathrm{G}$, was derived only from the father and was duplicated through UPiD.

Three possible chromosomal segregation patterns can be considered to explain this whole chromosomal UPiD (Fig. 3A). The first is monosomy rescue after fertilization of a nullisomic oocyte, and the second is fertilization by a disomic sperm and the elimination of the maternal chromosome by trisomy rescue. In this case, nondisjunction may have occurred during meiosis II during spermatogenesis with no crossing-over (normal gametogenesis and the consequences of nondisjunction are shown in online suppl. Fig. 1, 2, respectively). The third possibility is gamete complementation (GC), which refers to the fertilization of a nullisomic oocyte by a disomic sperm. Since GC requires 2 different abnormal gametogenesis events, it is considered a very rare event.

\section{Patient 2}

Mutational screening detected a missense mutation in ATPV0A4, NM_020632.2:c.1211T>C, NP_065683.2:p. (Leu404Pro) (Fig. 1B). Although this substitution has not been previously reported in the literature or database, in silico analysis revealed the pathogenicity of this substitu- 


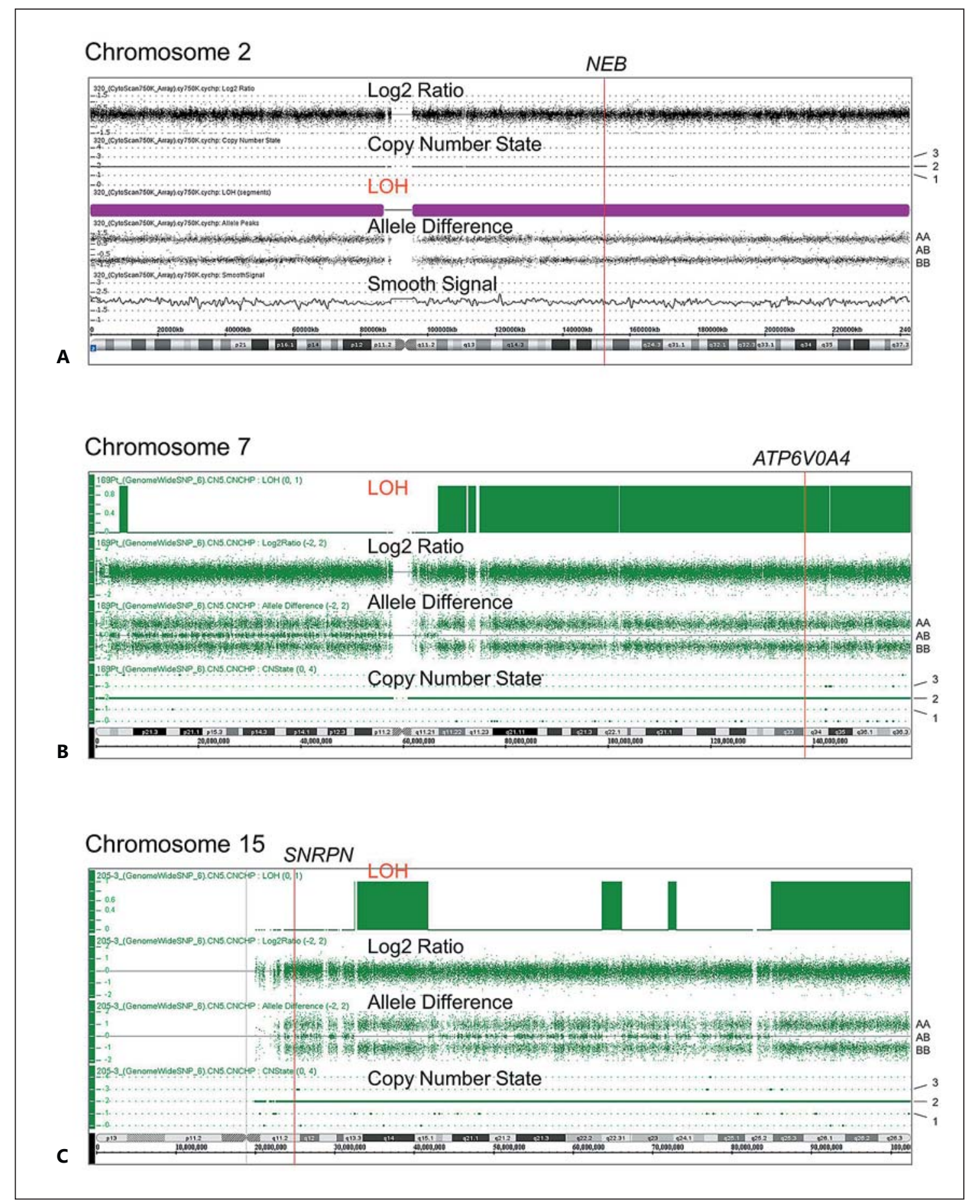

Fig. 2. SNP array results. Chromosomal regions of uniparental isodisomy (UPiD) are shown as copy number neutral $(=2)$ loss of heterozygosity $(\mathrm{LOH})$, and these regions do not include heterozygous SNP probe signals $(\mathrm{AB})$. In patient 1 , the entire chromosome 2 is the result of UPiD $(\mathbf{A})$. On the other hand, only the long arm of chromosome 7 is the result of UPiD in patient 2 (B), and segmental UPiD of chromosome 15 is seen in patient $3(\mathbf{C})$.

Uniparental Isodisomy Patterns that Cause Autosomal Recessive Disorders
Cytogenet Genome Res 2018;154:137-146 DOI: $10.1159 / 000488572$ 


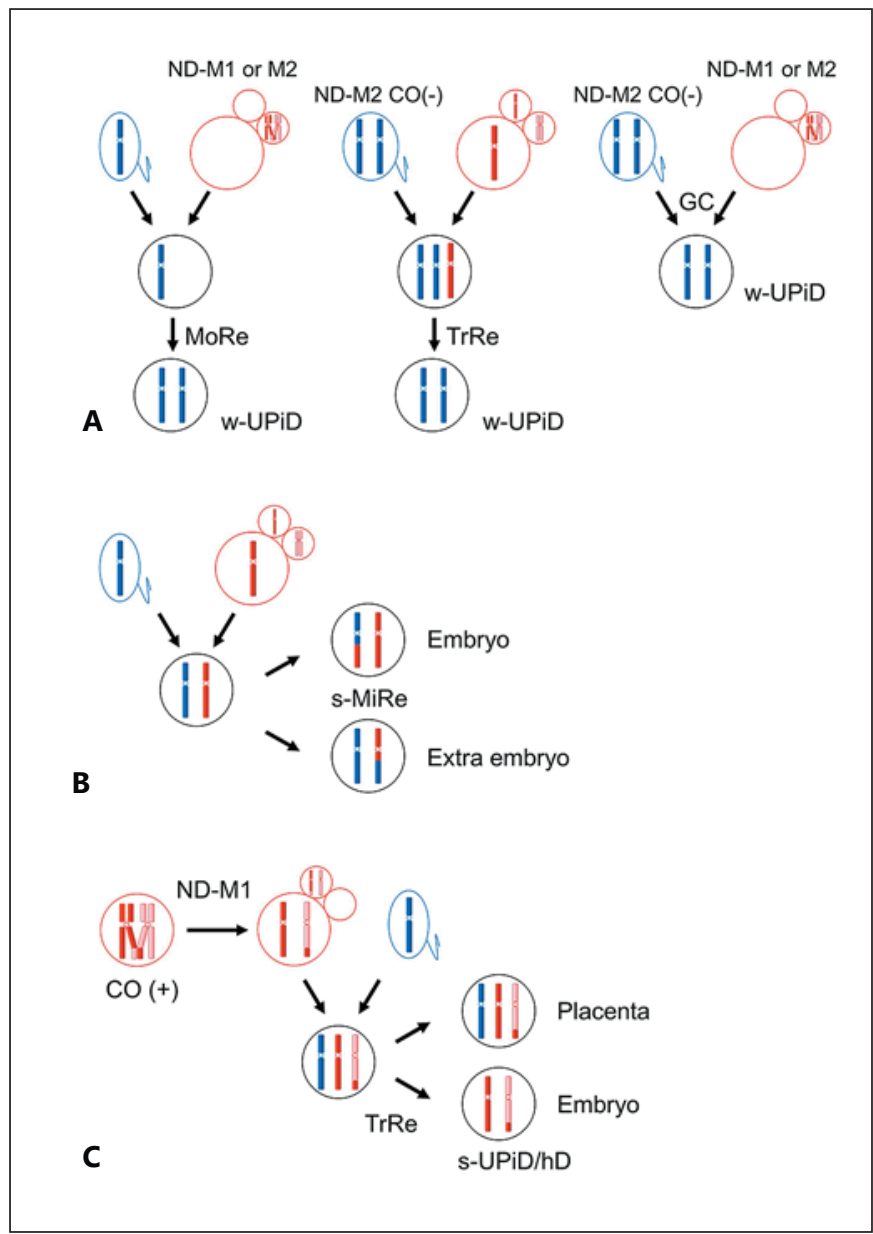

Fig. 3. Estimated events of gametogenesis and UPiD formation in patients 1 (A), 2 (B), and 3 (C). CO, crossing-over; GC, gamete complementation; M1, meiosis I; M2, meiosis II; MiRe, mitotic recombination; MoRe, monosomy rescue; $\mathrm{ND}$, nondisjunction; s-, segmental; TrRe, trisomy rescue; UPiD, uniparental isodisomy; $\mathrm{UPiD} / \mathrm{hD}$, uniparental isodisomy with heterodisomy; w-, whole.

tion. SIFT (http://sift.jcvi.org/) indicated an affected protein function with a score of 0.00 , Polyphen2 (http://genetics.bwh.harvard.edu/pph2/) showed the probability of damage with a score of 1.000 , and Pmut (http://mmb.pcb. ub.es/PMut/) predicted it to be pathological. We therefore concluded that this substitution is a novel ATPVOA4 mutation. Parental analysis revealed that the same missense mutation was present in the mother as a heterozygous carrier but not in the father (Fig. 1B). To determine whether the mutation was the result of paternal deletion or maternal UPiD, we performed a SNP array analysis (Fig. 2B). Interestingly, it revealed UPiD only in the long arm of chromosome 7, which includes the ATP6V0A4 locus. We concluded that the ATP6VOA4 mutation allele was derived only from the mother and duplicated through UPiD, which is a different chromosomal segregation mechanism than in patient 1. Maternal UPD7 is known to be one of the causative mechanisms of Silver-Russell syndrome (SRS, OMIM \#180860) and is identified in around $5-10 \%$ of cases [Wakeling, 2011]. In addition, several SRS patients with segmental maternal UPD7 have been reported, and the responsible segment is assumed to be located in the long arm of chromosome 7 [Eggermann et al., 2008], possibly at 7q31-qter [Hannula et al., 2001a]. We re-examined patient 2 at the age of 4 years and 9 months. His height was $89.8 \mathrm{~cm}(-3.6 \mathrm{SD})$, but short stature is also influenced by RTA. He did not show any motor developmental delay and was able to articulate sentences but showed some autistic features, such as social communication disturbance, hyperactivity, and hypersensitivity. Although micrognathia and clinodactyly of the fifth fingers were observed, a triangular face, hemihypertrophy and difference of lower limb length were not present. Overall, he manifested an incomplete SRS phenotype consistent with a UPD case [Hannula et al., 2001b; Wakeling, 2011].

In contrast to patient 1 , both paternal and maternal segments of the short arm of chromosome 7 were present but only the maternal copy of the long arm (online suppl. Table 2). Consequently, this event was considered to be the result of a post-zygotic mitotic recombination after normal fertilization, and the patient would have developed from one of the recombinant cells (Fig. 3B). This type of post-zygotic mitotic recombination is well known as one of the major mechanisms of the loss of heterozygosity of tumor suppressor genes in cancer [O'Keefe et al., 2010].

\section{Patient 3}

SNRPN methylation analysis detected amplified bands corresponding to both the methylated and unmethylated allele in the patient. However, the intensity of the unmethylated band was relatively faint (Fig. 1C). In this test, an unmethylated band was not observed in the PraderWilli syndrome sample, while a methylated band was not observed in the Angelman syndrome sample. Therefore, the results of the patient do not indicate a typical pattern of Prader-Willi syndrome. Subsequently, SNP array analysis was conducted (Fig. 2C), and segmental UPiD was detected in chromosome 15, but the PWS/AS region (the SNRPN locus) was located outside of the UPiD segments. Unlike in AR inherited Mendelian diseases, disorders caused by imprinted genes can also be the result of UPhD. We performed SNP array analysis of the parents to con- 


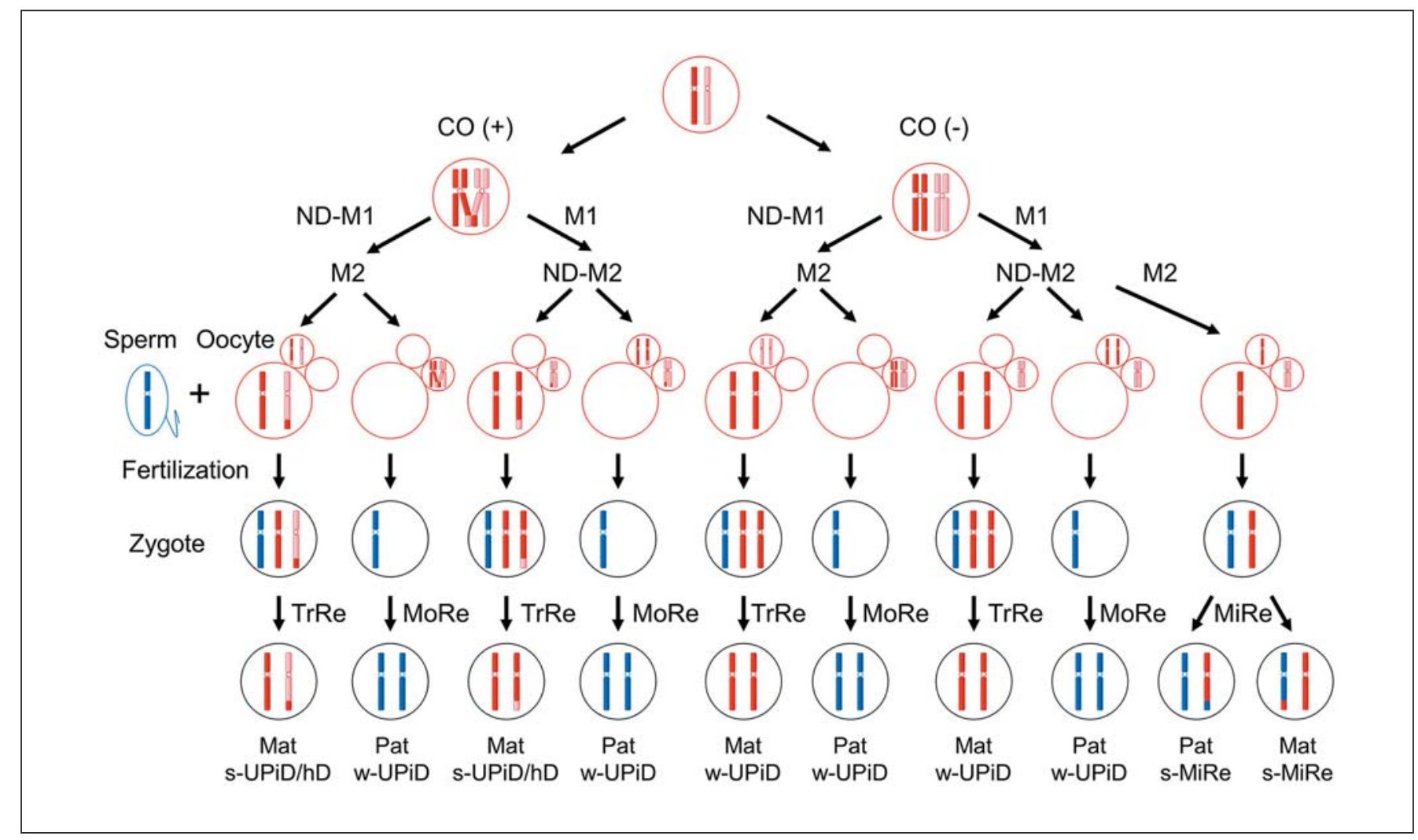

Fig. 4. All possible consequences of nondisjunction during oogenesis and subsequent UPiD formation. Mat, maternal; Pat, paternal. The other abbreviations are the same as for Figure 3.

firm segregation of the SNP markers on chromosome 15 to the patient. The results revealed that both chromosome 15 components of the patient were derived from the mother and did not involve any paternal origin (online suppl. Table 3). Some portions of chromosome 15 of the patient contained both maternal chromosomes 15 (UPhD), while other portions contained only 1 maternal chromosome 15 (UPiD). This segmental UPD could be explained by the nondisjunction of chromosome 15 during oogenesis after crossing-over (chiasma formation) and the elimination of the paternal chromosome 15 by trisomy rescue after fertilization (Fig. 3C). Whether nondisjunction of oogenesis occurred during meiosis I or II can be distinguished by whether the pericentromeric component is heterozygous (meiosis I) or homozygous (meiosis II) (online suppl. Fig. 2, 3). In this case, the pericentromeric area was the result of $\mathrm{UPhD}$, and consequently nondisjunction during meiosis I was considered, possibly accompanied by maternal age. The patient was suspected of confined placental mosaicism of trisomy 15 by fetal chromosomal analysis. The result of the methyla-

Uniparental Isodisomy Patterns that Cause Autosomal Recessive Disorders tion analysis indicated the presence of a small population of blood cells that contained a paternally-derived chromosome 15. However, GTG analysis of 100 metaphases did not detect any trisomy 15 cells.

Notably, if the UPiD portion of the chromosome contained a mutated gene, the patient could develop an AR disease according to a homozygous mutation as seen in patients 1 and 2. Indeed, there are several case reports of an AR disease caused by this type of abnormal chromosomal segregation (online suppl. Table 1).

\section{Discussion}

The first report on the development of an AR disease caused by UPiD was made by Spence et al. [1988], who described a patient with cystic fibrosis as a result of UPiD7. Subsequently, similar cases have been published, with the number of case reports increasing through the use of microsatellite analysis and SNP arrays which make it easier to analyze the parental origin

Cytogenet Genome Res 2018;154:137-146 143 DOI: $10.1159 / 000488572$ 
Table 3. Frequencies of reported UPiD patterns causing autosomal recessive diseases and the parental origin of the chromosome

\begin{tabular}{lccc}
\hline UPiD pattern & $\begin{array}{l}\text { Total, } \\
n(\%)\end{array}$ & $\begin{array}{l}\text { Paternal, } \\
n(\%)\end{array}$ & $\begin{array}{l}\text { Maternal, } \\
n(\%)\end{array}$ \\
\hline Segmental & $34(40.0)$ & $7(8.2)$ & $27(31.8)$ \\
$\quad$ MiRe & $7(8.2)$ & $3(3.5)$ & $4(4.7)$ \\
UPiD/hD & $27(31.8)$ & $4(4.7)$ & $23(27.1)$ \\
ND-M1 & $18(21.2)$ & $3(3.5)$ & $15(17.6)$ \\
ND-M2 & $9(10.6)$ & $1(1.2)$ & $8(9.4)$ \\
Whole chromosomal & $51(60.0)$ & $36(42.4)$ & $15(17.6)$ \\
\hline Total & $85(100)$ & $43(50.6)$ & $42(49.4)$ \\
\hline
\end{tabular}

M1, meiosis I; M2, meiosis II; MiRe, mitotic recombination; $\mathrm{ND}$, nondisjunction; $\mathrm{UPiD} / \mathrm{hD}$, uniparental isodisomy with heterodisomy.

of the chromosomes. Here, we reviewed the literature and collected 85 patients with AR disorders caused by UPiD in which the parental origin could be determined, including patients 1 and 2 of the present study. In reviewing the results of the literature, we realized that the patients could be classified into 3 major patterns, as demonstrated in the patients described here: whole chromosomal UPiD (w-UPiD), segmental UPiD through post-zygotic mitotic recombination (s-MiRe), and segmental UPiD with UPhD (s-UPiD/hD). Moreover, the last group could be subdivided by the timing of the nondisjunction at meiosis I (ND-M1) or meiosis II (ND-M2) by reviewing microsatellite analysis or SNP array data described in the literature (Table 3; online suppl. Table 1). Among the 85 cases, segmental UPiD accounted for 34 cases (40\%) (7 s-MiRe and 27s-UPiD/ $\mathrm{hD}$ ) and w-UPiD accounted for 51 cases (60\%). Overall, the parental origin of UPiD was evenly distributed, as 43 cases were of paternal origin and 42 were of maternal origin. However, interestingly, among the $51 \mathrm{w}$-UPiD patients, 36 were paternally and 15 were maternally derived. On the other hand, among the $27 \mathrm{~s}-\mathrm{UPiD} / \mathrm{hD}$ patients, 23 were of maternal origin and only 4 were of paternal origin. Of the 23 maternal s-UPiD/hD cases, 15 were classified as being ND-M1 and 8 being ND-M2. Of the $7 \mathrm{~s}$-MiRe patients, 3 were of paternal and 4 of maternal origin. The parental origin of the UPiD chromosome between the 3 groups clearly differed. All possible UPD segregation patterns are summarized in online suppl. Figure 3, and all UPiD patterns can be of either maternal or paternal origin. If the nondisjunction rate of spermatogenesis and oogenesis are the same, the parental origin of each pattern should approach parity.
It is reported that maternal UPD is more common than paternal UPD (1.6-3 times), and its major mechanism is disomic oocyte formation as a result of maternal age-related nondisjunction during meiosis I and subsequent trisomy rescue (elimination of the sperm-derived chromosome) after fertilization [Kotzot and Utermann, 2005; Liehr, 2010]. This process results in maternal UPhD and does not cause an AR disease immediately. However, when maternal homologous chromosomes undergo chiasma formation (crossing-over) during meiosis I, the same process results in s-UPiD/hD. On the other hand, when maternal homologous chromosomes did not show chiasma formation and nondisjunction during meiosis II, subsequent trisomy rescue results in maternal $\mathrm{w}$-UPiD. Nondisjunction of oogenesis both in meiosis I or II could produce a nullisomic oocyte. Subsequent monosomy rescue (the duplication of the sperm-derived chromosome) after fertilization results in paternal w-UPiD.

Considering nondisjunction during oogenesis as a starting point, it is possible to explain the different proportions and parental origins in each UPiD pattern. As shown in Figure 4, a nullisomic oocyte results in paternal w-UPiD through monosomy rescue. On the other hand, a disomic oocyte results in both $\mathrm{s}-\mathrm{UPiD} / \mathrm{hD}$ with crossing-over and maternal $\mathrm{w}$-UPiD without crossing-over through trisomy rescue. Overall, w-UPiDs are created in a majority, with both parental origins but a paternal dominancy. Segmental UPiD constitutes a smaller population in which the majority of s-UPiD/hD is of maternal origin, while s-MiRe show an equal frequency of parental origin. This model is in good agreement with the observed frequencies in Table 3. Although the literature cases collected here do not represent a real cohort, this model reasonably explains why the ratio of the parental origin is different in each pattern and seems to represent the framework of reality. The small population of paternal s-UPiD/hD reflects the low frequency of nondisjunction during spermatogenesis. Moreover, as shown in Table 3, w-UPiD accounts for the majority of cases, and the frequency of paternal w-UPiD is roughly twice than that of maternal w$\mathrm{UPiD}$, while the relatively rare event s-MiRe has an equal parental origin. These results are consistent with those expected from Figure 4.

Advanced maternal age is known to be a risk factor for the occurrence of nondisjunction [Jones, 2008]. Therefore, it is considered that the occurrence of AR disorders by UPiD also correlates with high maternal age. In our cases, the mothers of patients 1 and 2 were 36 and 34 years old, respectively, when they gave birth. Unfortunately, there was almost no information on maternal age for the

Niida/Ozaki/Shimizu/Ueno/Tanaka 
patients described in the literature, and, therefore, no conclusion could be drawn. On the other hand, there are several lines of evidence showing a correlation between UPD and maternal age [Matsubara and Ogata, 2013]. In the studies of Prader-Willi syndrome, a positive relationship between advanced maternal age and the occurrence of maternal UPD15 was reported [Kotzot, 2004; Whittington et al., 2007; Cho et al., 2013]. The authors explained this to be the result of the generation of a disomic 15 oocyte resulting from the age-dependent risk of nondisjunction during oogenesis and that consecutive trisomy rescue occurred after fertilization. In addition, a positive association of advanced maternal age with paternal UPD14 in Japanese patients has been reported. The authors interpreted this as the result of a higher maternal age, which in this case also raised the risk of generating a nullisomic 14 oocyte, following which a consecutive monosomy rescue produced upd(14)pat [Kagami et al., 2012]. In this way, advanced maternal age is thought to raise the probability of generating both paternal and maternal UPiD through the age-related risk of nondisjunction during oogenesis (Fig. 4).

The natural frequency of UPD is still not known. One of the best estimates can be drawn from the incidence of imprinting disorders. For example, the frequency of UPD15, the most well-known UPD causing Prader-Willi and Angelman syndrome, is estimated to occur in $1 / 80,000$ of all births. If we assume that the frequency of UPD for other chromosomes is the same, then UPD for any chromosome can be expected in 1/3,500 births $(1 / 80,000 \times 23$ chromosomes $)$ [Liehr, 2010; Yamazawa et al., 2010]. However, this discussion does not take into account the natural abortion rate of upd(15). Also, one should consider the existence of chromosomes without imprinting genes which UPD does not affect phenotype and abortion rate. Thus, there is a possibility of underestimation. For many chromosomes (1-5, 8-10, 12, 13, 17$19,21,22, \mathrm{X})$, there are no known UPD-related diseases, whereas normal phenotypes are reported in UPD 1-4, 8 , $12,13,18$, and 21 (OMIC TOOLS, UPD; http://upd-tl. com/upd.html). Moreover, another major problem is that the frequency of UPD varies depending on the size of the chromosomal segment. Smaller segments of chromosomes increase the frequency of UPiD [Sasaki et al., 2013] but reduce the chance of causing an AR disease, as the probability that the segment includes a disease causing gene is reduced.

In our own experience, we encounter AR diseases caused by UPiD every 2-3 years [Niida et al., 2012b, 2016]. We test various AR diseases, and approximately

Uniparental Isodisomy Patterns that Cause Autosomal Recessive Disorders
$10-15$ tests are performed per year. Although it is not possible to calculate accurate frequencies, it seems certain that there are more cases than previously thought. In terms of practice, it is important to keep in mind that the incidence of UPiD is lower in AR disorders with a high frequency in the population (i.e., high frequency of disease carriers). Conversely, for rare AR disorders, the possibility of both parents being carriers and the incidence of UPiD approaches parity when the patient has a homozygous mutation and the parents are non-consanguineous. The family of a patient should be informed of this fact during genetic counselling before genetic testing, as in the case of UPiD the recurrence risk for siblings is negligible. Accurate diagnosis provided by SNP array is the standard strategy today, and knowing that there are 3 patterns of the mechanisms of UPiD that produce AR disorders is important in order to correctly interpret the test results. Recent innovation has been impressive and SNP arrays may be superseded by next-generation sequencing in the near future [Wang et al., 2014].

\section{Acknowledgements}

The authors thank Dr. Ichizo Nishino, National Center of Neurology and Psychiatry, Tokyo, for the initial genetic screening test of the patient 1 . Financial support for this study was provided by KAKENHI under grant number 16K08980 and AMED under grant number 17kk0205012s1202.

\section{Statement of Ethics}

For all 3 patients, written informed consent was obtained from the parents after genetic counselling. The study design was approved by the Ethics Review Board of Kanazawa Medical University.

\section{Disclosure Statement}

The authors have no conflicts of interest to declare.

Batlle D, Ghanekar H, Jain S, Mitra A: Hereditary
distal renal tubular acidosis: new understand-
ings. Annu Rev Med 52:471-484 (2001).
Cho SY, Ki CS, Sohn YB, Maeng SH, Jung YJ, et
al: The proportion of uniparental disomy is
increased in Prader-Willi syndrome due to an
advanced maternal childbearing age in Korea.
J Hum Genet 58:150-154 (2013).
Eggermann T, Schönherr N, Jäger S, Spaich C,
Ranke MB, et al:Segmental maternal UPD(7q)
in Silver-Russell syndrome. Clin Genet 74:
486-489 (2008).

Cytogenet Genome Res 2018;154:137-146 145 
-Hannula K, Lipsanen-Nyman M, Kontiokari T, Kere J: A narrow segment of maternal uniparental disomy of chromosome 7q31-qter in Silver-Russell syndrome delimits a candidate gene region. Am J Hum Genet 68:247-253 (2001a).

-Hannula K, Kere J, Pirinen S, Holmberg C, Lipsanen-Nyman M: Do patients with maternal uniparental disomy for chromosome 7 have a distinct mild Silver-Russell phenotype? J Med Genet 38:273-278 (2001b).

-Jones KT: Meiosis in oocytes: predisposition to aneuploidy and its increased incidence with age. Hum Reprod Update 14:143-158 (2008).

-Kagami M, Kato F, Matsubara K, Sato T, Nishimura G, Ogata T: Relative frequency of underlying genetic causes for the development of UPD(14)pat-like phenotype. Eur J Hum Genet 20:928-932 (2012).

-Kosaki K, McGinniss MJ, Veraksa AN, McGinnis WJ, Jones KL: Prader-Willi and Angelman syndromes: diagnosis with a bisulfite-treated methylation-specific PCR method. Am J Med Genet 73:308-313 (1997).

Kotzot D: Advanced parental age in maternal uniparental disomy (UPD): implications for the mechanism of formation. Eur J Hum Genet 12:343-346 (2004).

Kotzot D, Utermann G: Uniparental disomy (UPD) other than 15: phenotypes and bibliography updated. Am J Med Genet A 136: 287-305 (2005).
Lapunzina P, Monk D: The consequences of uniparental disomy and copy number neutral loss-of-heterozygosity during human development and cancer. Biol Cell 103:303-317 (2011).

Liehr T: Cytogenetic contribution to uniparental disomy (UPD). Mol Cytogenet 3:8 (2010).

- Matsubara K, Ogata T: Advanced maternal age at childbirth and the development of uniparental disomy. A commentary on the proportion of uniparental disomy is increased in PraderWilli syndrome due to an advanced maternal childbearing age in Korea. J Hum Genet 58: 118-119 (2013).

Niida Y, Kuroda M, Mitani Y, Okumura A, Yokoi A: Applying and testing the conveniently optimized enzyme mismatch cleavage method to clinical DNA diagnosis. Mol Genet Metab 107:580-585 (2012a).

Niida Y, Kuroda M, Mitani Y, Yokoi A, Ozaki M: Paternal uniparental isodisomy of chromosome 22 in a patient with metachromatic leukodystrophy. J Hum Genet 57:687-690 (2012b).

Niida Y, Ozaki M, Inoue M, Takase E, Kuroda M, et al: CHIPS for genetic testing to improve a regional clinical genetic service. Clin Genet 88:155-160 (2015).

Niida Y, Yokoi A, Kuroda M, Mitani Y, Nakagawa $\mathrm{H}$, Ozaki M: A girl with infantile neuronal ceroid lipofuscinosis caused by novel PPT1 mutation and paternal uniparental isodisomy of chromosome 1. Brain Dev 38:674-677 (2016).

-O'Keefe C, McDevitt MA, Maciejewski JP: Copy neutral loss of heterozygosity: a novel chromosomal lesion in myeloid malignancies. Blood 115:2731-2739 (2010).
Sasaki K, Mishima H, Miura K, Yoshiura K: Uniparental disomy analysis in trios using genome-wide SNP array and whole-genome sequencing data imply segmental uniparental isodisomy in general populations. Gene 512: 267-274 (2013).

- Sharma S, Gupta A, Saxena S: Comprehensive clinical approach to renal tubular acidosis. Clin Exp Nephrol 19:556-561 (2015).

- Spence JE, Perciaccante RG, Greig GM, Willard HF, Ledbetter DH, et al: Uniparental disomy as a mechanism for human genetic disease. Am J Hum Genet 42:217-226 (1988).

- Tian X, Liang WC, Feng Y, Wang J, Zhang VW, et al: Expanding genotype/phenotype of neuromuscular diseases by comprehensive target capture/NGS. Neurol Genet 1:e14 (2015).

Wakeling EL: Silver-Russell syndrome. Arch Dis Child 96:1156-1161 (2011).

-Wang Y, Li W, Xia Y, Wang C, Tang YT, et al: Identifying human genome-wide $\mathrm{CNV}, \mathrm{LOH}$ and UPD by targeted sequencing of selected regions. PLoS One 10:e0123081 (2014).

-Whittington JE, Butler JV, Holland AJ: Changing rates of genetic subtypes of Prader-Willi syndrome in the UK. Eur J Hum Genet 15:127130 (2007).

- Yamazawa K, Ogata T, Ferguson-Smith AC: Uniparental disomy and human disease: an overview. Am J Med Genet C Semin Med Genet 154C:329-334 (2010). 